\title{
Notable Stabilization of $\alpha$-Chymotrypsin by the Protic Ionic Additive, [ch][dhp]: Calorimetric Evidence for a Fine Enthalpy/Entropy Balance
}

\author{
Sophio Uchaneishvili, ${ }^{1}$ Maya Makharadze, ${ }^{1}$ Mikhael Shushanyan, ${ }^{1,2}$ \\ Rudi van Eldik, ${ }^{3}$ and Dimitri E. Khoshtariya ${ }^{1,2,3}$ \\ ${ }^{1}$ Department of Biophysics, I. Beritashvili Center of Experimental Biomedicine, Gotua 14, 0160 Tbilisi, Georgia \\ ${ }^{2}$ Institute for Biophysics and Bionanosciences at the Department of Physics, I. Javakhishvili Tbilisi State University, \\ I. Chavchavadze Avenue 3, 0128 Tbilisi, Georgia \\ ${ }^{3}$ Department of Chemistry and Pharmacy, Friedrich-Alexander University of Erlangen-Nürnberg, Egerlandstraße 1, \\ 91058 Erlangen, Germany
}

Correspondence should be addressed to Dimitri E. Khoshtariya; dimitri.khoshtariya@tsu.ge

Received 3 March 2014; Accepted 23 June 2014; Published 7 September 2014

Academic Editor: Monir Moniruzzaman

Copyright (C) 2014 Sophio Uchaneishvili et al. This is an open access article distributed under the Creative Commons Attribution License, which permits unrestricted use, distribution, and reproduction in any medium, provided the original work is properly cited.

\begin{abstract}
An impact of 0.5 to $3 \mathrm{M}$ choline dihydrogen phosphate, [ch][dhp], the biotechnologically relevant ionic substance, on the thermal stability of a model globular protein, $\alpha$-chymotrypsin $(\alpha$-CT), has been studied exploiting the highly sensitive differential scanning calorimetry (DSC) technique. The notable overall stabilizing effect of $11 \pm 2 \mathrm{~K}$ regarding the thermal transition (melting) temperature, $T_{m}$, has been detected. For this kind of series, for the first time, the calorimetric melting enthalpy $\left(\Delta H_{\text {cal }}\right)$ and transition entropy $\left(\Delta S_{m}\right)$ parameters have been determined simultaneously throughout. The first analysis indicated a two-phase impact implying (a) the initial, dramatic drop in both $\Delta H_{\text {cal }}$ and $\Delta S_{m}$, obviously connected to specific, direct interaction between the [ch][dhp] components and $\alpha$-CT's charged groups (within 0 to $1 \mathrm{~mol} / \mathrm{L}$ [ch][dhp]), leading to the essential rearrangement of the interfacial hydrogen-bonded (HB) network; and (b) the follow-up (within 1 to $3.0 \mathrm{~mol} / \mathrm{L}$ [ch] [dhp]), modest changes in $\Delta H_{\text {cal }}$ and lack of changes in $\Delta S_{m}$, seemingly connected with a subsequent steady strengthening of already reformed HB network, respectively. These changes, presumably, are primarily facilitated by Coulombic interactions between the [dhp] anions and solvent-exposed positively charged amino groups of $\alpha$-CT.
\end{abstract}

\section{Introduction}

Currently, there is a great interest in the multipurpose application of recently revived substances, organic salts possessing relatively low melting points (also known as "roomtemperature ionic liquids," RTILs, or "plastic ionic crystals"). These substances, when mixed with water, are able to form a huge amount of unique liquid or semisolid compositions apt for the novel nanotechnological and bionanotechnological developments [1-4]. Because of virtually unlimited possibilities for the variation of an intrinsic molecular balance between the charged and uncharged (hydrophobic) groups on both positive and negative ionic components [1], these substances may offer potentially ideal milieu for any kind of reactant species including biomolecules (e.g., globular proteins), assembled in any kind of (bio)nanodevice. For the latter case, the major point, actually, implies design of optimal environmental conditions for the improved stability, activity, and refolding ability of "working" elements such as proteins, for example [4-22]. Furthermore, introduction of protic RTILs, thanks to their essential protonic conductivity, offers superior conditions for the proper functioning of bioinspired nanovoltaic cells/devices [1,3,22].

As to the stability issue for globular proteins under the impact of different additives, including RTILs, it can be adequately approached by the method of differential scanning 
calorimetry (DSC) $[4,5,8-11,13-17,23-26]$. Specifically, the DSC technique detects the temperature-induced unfolding (melting) process (associated with a global transition along the protein's collective conformational coordinate) as a variation of the protein's partial heat capacity versus temperature and, in uncomplicated cases, in a model-free way (directly) determines the unique "transition temperature" (temperature of thermal melting), $T_{m}$, the unfolding (melting) calorimetric enthalpy, $\Delta H_{\text {cal }}$ (i.e., the heat absorbed through the unfolding transformation around $T_{m}$ ), and calorimetric entropy, $\Delta S_{\text {cal }}$ [27-29].

Numerous papers on DSC studies for the stabilizing/destabilizing impact of various organic additives $[4,5,8$ $11,13-17,23-26,30]$, including RTILs [4, 5, 8-11, 13-17], on the proteins' global stability have been published. However, because of a complex, dualistic character of the RTIL impact (due to the involvement of oppositely charged surfaceactive ions), notwithstanding of several serious attempts [16, $17,21]$, no conclusive rationalization of the existing data was drawn so far. Obviously, more systematic and careful work with the application of the DSC combined with other methodologies is required. Indeed, in many cases of serial DSC studies implying the RTIL impact, typically, only the parameter $T_{m}$, as a measure of stability, has been considered; the easily calculable parameter $\Delta H_{\text {cal }}$ has been addressed in very few cases (see, e.g., $[5,8,9]$; for other similar studies involving uncharged additives, see, e.g., $[4,23,26,30])$. It is instructive that another easily calculable parameter, $\Delta S_{\text {cal }}$ (vide infra), has never been implicated for this kind of series. An entropic contribution is equally important for the understanding of the protein stability phenomenon, as its enthalpic counterpart $[29,31-33]$. In fact, stability of the native state results from the fine balance between a multitude of the enthalpic and entropic contributing factors (vide infra) $[4,27,30,31,33]$. Among other model proteins, in which thermal stability has been extensively explored by the DSC technique, $\alpha$-chymotrypsin ( $\alpha$-CT, EC 3.4.21.1; $M_{r}=26,000$ ), a hydrolytic enzyme of biotechnological importance, should be mentioned $[4,5,23,24,26,30]$. However, detailed studies regarding the impact of RTILs on $\alpha$-CT, using highly sensitive DSC technique (implying determination and analysis of all the key parameters, $T_{m}, \Delta H_{\text {cal }}$, and $\Delta S_{\text {cal }}$ ), are still lacking. In this communication, we report preliminary data and principal thermodynamic analysis on the stabilizing impact of biotechnologically important concentrations of a protic ionic salt, choline dihydrogen phosphate, $[\mathrm{ch}][\mathrm{dhp}]$, on the thermal stability of model protein, $\alpha$-CT. Determination of both enthalpic and entropic components contributing to the protein melting process allowed for a conjecturing of fine interfacial effects behind the notable stabilization impact.

\section{Experiment}

Highly purified and lyophilized $\alpha$-CT was purchased from Fluka and [ch][dhp] was from Iolitech, $\mathrm{GmbH}$, and used without further purification. Other chemicals were of highest purity available. The $\alpha$-CT samples for the DSC experiments

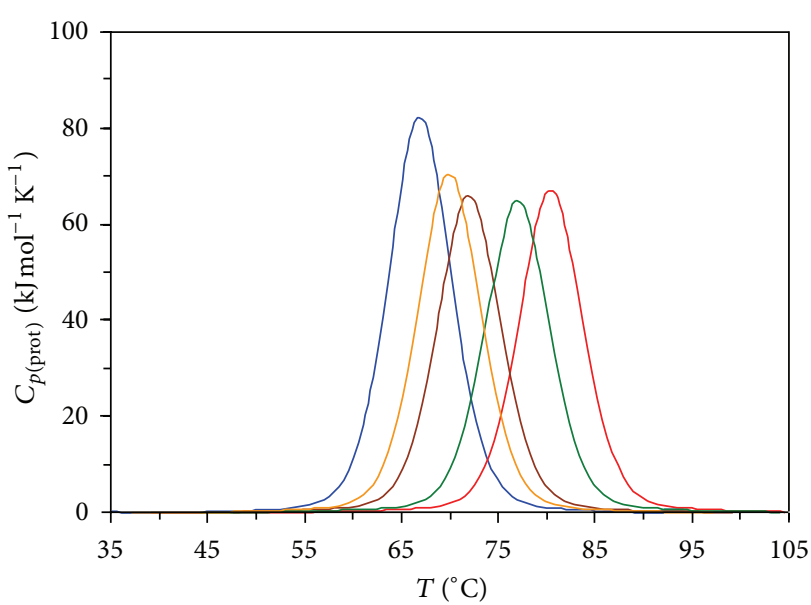

FIGURE 1: The DSC curves for the $\alpha$-CT thermal unfolding in the absence and presence of 0.5 to $3 \mathrm{M}$ [ch][dhp] concentrations; $\mathrm{pH}$ 4.2 (phosphate buffer).

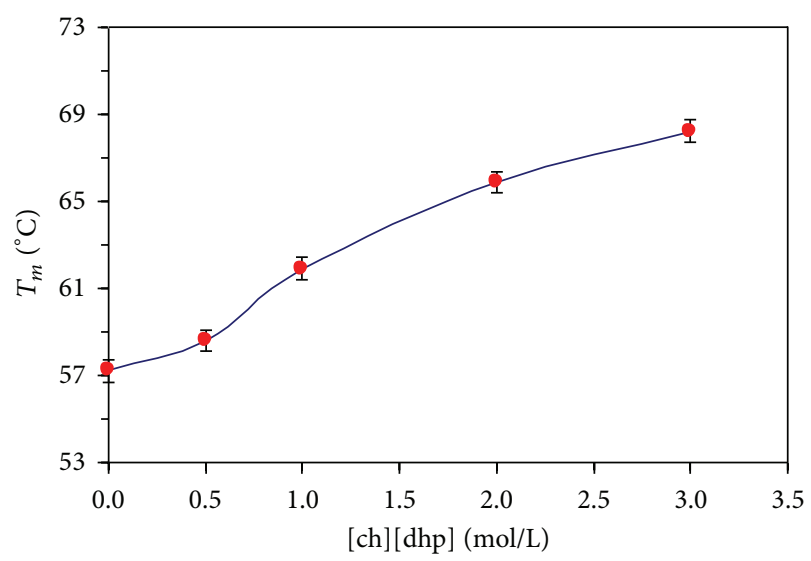

FIGURE 2: Dependencies of the transition temperatures for the temperature-induced melting of $\alpha$-CT on the [ch] [dhp] concentration (0 to $3 \mathrm{M} ; \mathrm{pH} 4.2$ ).

were prepared at concentrations of $1.5 \mathrm{mg} / \mathrm{mL}$, by dissolving in phosphate $10^{-2} \mathrm{M}$ buffer solutions ( $\mathrm{pH} 4.2$ ).

Microcalorimetric measurements for temperatureinduced melting (denaturation) of $\alpha$-CT in buffered [ch] [dhp] aqueous solutions were performed with a DASM$4 \mathrm{~A}$ adiabatic scanning calorimeter (Biopribor, Russia) integrated with a PCI-DAS1001 (Measurement Computing Corporation) interface unit, providing direct PC access [30]. The heating rate was $2 \mathrm{~K} / \mathrm{min}$. The methodological aspects of the DSC technique and data processing have been described earlier $[23,27,28,30]$.

\section{Results and Discussion}

Figure 1 depicts the zero-baseline-corrected calorimetric curves (partial heat capacity of protein versus temperature) for the temperature-induced melting of $\alpha$-CT in the absence and presence of the $[\mathrm{ch}][\mathrm{dhp}]$ additives, $0,0.5,1.0,2.0$, and $3.0 \mathrm{~mol} / \mathrm{l}$. Accordingly, Figures 2, 3, and 4 and Table 1 depict 


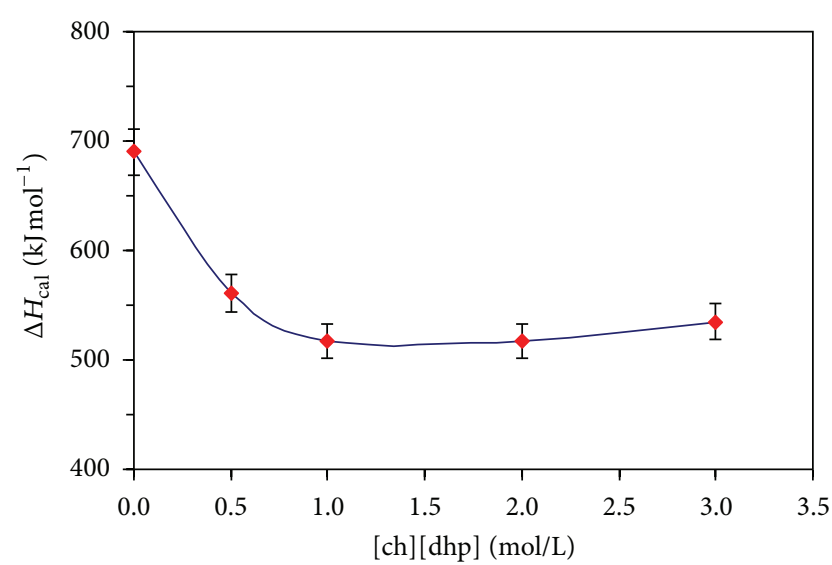

FIGURE 3: Dependence of the calorimetric enthalpy for temperatureinduced melting of $\alpha$-CT on the [ch] [dhp] concentration (0 to $3 \mathrm{M}$; $\mathrm{pH}$ 4.2).

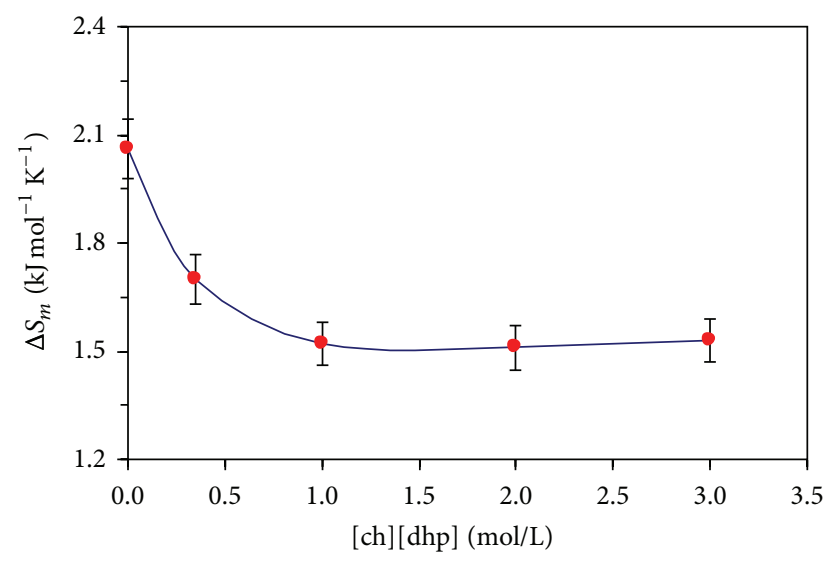

FIGURE 4: Dependence of the transition entropy for temperatureinduced melting of $\alpha$-CT on the [ch] [dhp] concentration (0 to $3 \mathrm{M}$; $\mathrm{pH}$ 4.2).

TABLE 1: Thermodynamic parameters for the thermal unfolding of the $\alpha$-CT in the presence of different [ch][dhp] concentrations phosphate buffer, $\mathrm{pH} 4.2$.

\begin{tabular}{lcccc}
\hline $\begin{array}{l}\text { ch] [dhp], } \\
\text { mol/L }\end{array}$ & $\begin{array}{c}{[\mathrm{ch}][\mathrm{dhp}],} \\
\%(\mathrm{w} / \mathrm{w})\end{array}$ & $\begin{array}{c}T_{m},{ }^{\circ} \mathrm{C} \\
(\mathrm{K})\end{array}$ & $\begin{array}{c}\Delta H_{\mathrm{cal}}, \\
\mathrm{kJ} \mathrm{mol}^{-1}\end{array}$ & $\begin{array}{c}\Delta S_{m}, \\
\mathrm{~kJ} \mathrm{~mol}^{-1} \mathrm{~K}^{-1}\end{array}$ \\
\hline 0 & 0 & $62.8(335.8)$ & 690 & 2.06 \\
0.5 & 12.5 & $65.8(338.8)$ & 560 & 1.70 \\
1 & 25 & $67.7(340.7)$ & 517 & 1.52 \\
2 & 50 & $72.1(345.1)$ & 520 & 1.51 \\
3 & 75 & $76.0(349.0)$ & 535 & 1.53 \\
\hline
\end{tabular}

results of thermodynamic analysis of these melting curves. The values for a melting enthalpy, $\Delta H_{\text {cal }}$, were determined as areas under the calorimetric curves of Figure $1[23,27-30]$ :

$$
\Delta H_{\text {cal }}=\int_{T_{1}}^{T_{2}} C_{p(\text { prot })} d T,
$$

where $T$ is the absolute temperature and $T_{1}$ and $T_{2}$ are the temperatures that correspond to the start and completion of heat absorption due to the protein's thermal melting $\left(T_{1}<\right.$ $T_{m}<T_{2}$ ). The values of the counterpart parameter, melting entropy, $\Delta S_{\text {cal }}$, can be determined as follows [29]:

$$
\Delta S_{\text {cal }}=\int_{T_{1}}^{T_{2}} \frac{C_{p(\text { prot })}}{T} d T .
$$

Under the assumption that a two-state model for the protein melting process is operative, that is, (a) at $T_{m}$ concentrations of the native and melted (unfolded) protein are equal and (b) virtually the entire entropy change takes place at this temperature (therefore, $\Delta G_{m}=\Delta H_{\text {cal }}-T_{m} \Delta S_{m}=0$ ), (2) is simplified as follows:

$$
\Delta S_{m}=\frac{\Delta H_{\text {cal }}}{T_{m}} .
$$

However, one may question whether (1) to (3) and related analysis (vide infra) are valid for the case of $\alpha$-CT, known as a protein in which thermal unfolding process, in general, deviates from a simple two-state pattern (see, e.g., $[24,30])$. Rather detailed analysis of this issue is given in our previous paper [30] (see also [17, 34]). In brief, in the course of a thermal melting of most globular proteins of modest dimensions, including $\alpha$-CT, the native and "normally" unfolded states can be considered as yet dominantly populated states against the relatively minor, thermally induced molten globule (predenatured) [25, 34], "intermediate" (metastable transitional) [16, 34], and misfolded/selfaggregated (postdenatured) states $[16,29,35]$. Importantly, the DSC curves retained symmetrical, nearly Gaussian shape (half widths were within $6.7 \pm 0.2 \mathrm{~K}$ throughout) upon the addition of 0.5 to $3 \mathrm{~mol} / \mathrm{l}$ [ch] [dhp] (the fact that favors the abovementioned outline, yet, as a crude approximation [30]), whilst their maximum position $\left(T_{m}\right)$ was gradually shifted to higher temperatures (for ca. $11 \mathrm{~K}$, in overall), pointing to the notable stabilizing impact of [ch][dhp] (Table 1). The values of $\Delta S_{\text {cal }}$ (attained for selected cases) and $\Delta S_{m}$, as determined through (2) and (3), coincided within the limits of experimental error, that is, an additional support for the practical applicability of (3). The slope for the first change of $T_{m}$ versus the [ch][dhp]) content (from 0 to $1 \mathrm{~mol} / \mathrm{l}$ [ch][dhp]) was a little bit larger than one for the rest of the concentration range (Figure 2). This fact could be ascribable to the experimental scatter of experimental points if there is no remarkable and drastic mutually compensating change of both $\Delta H_{\text {cal }}$ and $\Delta S_{m}$ resulting only in a modest shift of $T_{m}$ (Figures 2 to 4, Table 1). Next, in a concentration range from 1 to $3 \mathrm{~mol} / \mathrm{l}[\mathrm{ch}][\mathrm{dhp}]$, parameter $\Delta H_{\text {cal }}$ increased gradually very little, whereas parameter $\Delta S_{m}$ remained unchanged within the limits of experimental error. This occurrence allowed parameter $T_{m}$ to increase further steadily (Figure 2) (vide infra).

Interestingly, Constatinescu et al. [11] recently reported on ca. $16 \mathrm{~K}$ increase in the melting temperature of another model protein, ribonuclease A, upon the adding of $3 \mathrm{~mol} / \mathrm{L}$ [ch] [dhp] (overall, $\approx 20 \mathrm{~K}$ increase upon the adding of $4 \mathrm{~mol} / \mathrm{L}$ [ch][dhp]). The shift values for $T_{m}$ and a trend for the 
dependence of $T_{m}$ on the [ch] [dhp] content are very similar to ours, having the distinct slope break at $0.5 \mathrm{~mol} / \mathrm{L}$ (see Figure 2 of [11]). However, as can be judged based on the incomplete Figure 1 of [14], the increase in $T_{m}$ was accompanied by the substantial increase in $\Delta H_{\text {cal }}$. Unfortunately, the whole trend for $\Delta H_{\text {cal }}$ (and, even more so, for $\Delta S_{m}$ ) is not reported in [11]. In another recent work, Rodrigues et al. [15] reported on ca. $11 \mathrm{~K}$ increase in a melting temperature of the third model protein, lysozyme, upon the adding of $2.5 \mathrm{~mol} / \mathrm{L}$ [ch][dhp]. The overall effect is absolutely similar to ours (implying the interpolated value of $T_{m} \approx 74^{\circ} \mathrm{C}$ at $2.5 \mathrm{~mol} / \mathrm{L}$ [ch] [dhp] for our system); however, the overall dependence of $T_{m}$ on the [ch][dhp] content for lysozyme, in contrast to our case (Figure 1), exhibited well-pronounced minimum at $0.5 \mathrm{~mol} / \mathrm{L}$ [ch] [dhp]. Unfortunately, again, the authors of [15] did not report the values for $\Delta H_{\text {cal }}$ and $\Delta S_{\text {cal }}\left(\Delta S_{m}\right)$ at any concentration of the additive. The lacking information would allow one to see the variable balance between $\Delta H_{\text {cal }}$ and $\Delta S_{m}$ behind the resultant behavior of $T_{m}$ in these studies, hence, to compare the complete data for all three model proteins and gain better insight into atomistic mechanisms of stabilization.

Nonetheless, the first analysis of our results together with comparable published data $[11,15]$ permits presuming that the mechanisms behind the initial drop in values of both $\Delta H_{\text {cal }}$ and $\Delta S_{m}$ and further slight increase with and virtual independence of $T_{m}$, respectively, are obviously different. When going from zero to $0.5 \div 1 \mathrm{~mol} / \mathrm{L}$ additive concentration, seemingly, strong association between the [ch][dhp] components and proteins' charged groups takes place that should be rather specific to the protein entity and its ionization state $(\mathrm{pH})$; note different patterns of $T_{m}$ for $\alpha$-CT and ribonuclease A on the one hand and lysozyme on the other hand (note also different $\Delta H_{\text {cal }}$ patterns for $\alpha$-CT and ribonuclease A, mentioned above). Around this point of the additive concentration, the specific interactions, probably, arrive at the saturation level, after which more gradual/monotonic changes of $T_{m}$ (in all three cases) and very small/no variation of $\Delta H_{\text {cal }}$ and $\Delta S_{m}$ (as detected in our case) can be observed. The small changes in $\Delta H_{\text {cal }}$, seemingly, are connected with the formation of additional contacts and some redistribution of existing ones between the protein and the [ch][dhp] components. Development of the latter process seems to favor proteins' gradual stabilization via the $\Delta H_{\text {cal }}$ rather than the $\Delta S_{m}$ parameter. According to the analysis of [16] (the most systematic one, so far), the choline cation is deemed to act as a slightly destabilizing component whereas dihydrogen phosphate anion is known as an efficient stabilizer. In addition, this pattern turned to be dependent on the additive concentration and has to be also essentially $\mathrm{pH}$ dependent [16]. Hence, around $\mathrm{pH} 4.2$, where the exposed amino groups are $100 \%$ positively charged, the [dhp] anions, able to effectively interact with these groups through the Coulombic attraction, may eventually replace the existing hydrogen-bond networks originally formed by solvating water and contribute to the protein stabilization. The enthalpic rather entropic nature of the stabilization impact of [ch][dhp] above $1 \mathrm{~mol} / \mathrm{L}$ concentration definitely points to a prevailing role of the hydrogen-bond network strengthening rather than that of additional strengthening of electrostatic interactions (between any kind of the "point charges" involved). At this $\mathrm{pH}$, the surface exposed carboxylate groups of $\alpha$-CT are for only ca. $50 \%$ negatively charged; therefore, respective Coulombic and associated hydrogenbonding interactions may play relatively minor role. Further systematic work is in progress aiming at more comprehensive insights at the atomistic level.

\section{Conclusions}

(a) We applied the very sensitive DSC technique to study an impact of 0.5 to $3 \mathrm{~mol} / \mathrm{L}$ additives of the biotechnologically relevant ionic substance [ch][dhp] on the thermal stability of a model globular protein $\alpha$-CT and found the notable stabilizing effect of $11 \pm 2 \mathrm{~K}$ regarding the thermal unfolding (melting) temperature, $T_{m}$, that varied monotonically with the additive concentration.

(b) The calorimetric melting enthalpy $\left(\Delta H_{\text {cal }}\right)$ and transition entropy $\left(\Delta S_{m}\right)$ parameters were determined simultaneously throughout the series, and the trend analysis indicated a two-phase pattern including the low-concentration (0 to $1 \mathrm{~mol} / \mathrm{L}[\mathrm{ch}][\mathrm{dhp}]$ ) and high-concentration (1 to $3 \mathrm{~mol} / \mathrm{L}$ [ch][dhp])) regions, connected to the initial dramatic and the follow-up minor or no changes of $\Delta H_{\text {cal }}$ and $\Delta S_{m}$, respectively.

(c) The atomistic mechanism behind the observed overall stabilizing effect, presumably, is guided by the ion pair formation between the protein's charged groups and the [ch] [dhp] components, with a prevailing role of restructured hydrogen-bonded networks facilitated mostly by Coulombic interaction between the [dhp] anions and solvent-exposed positively charged amino groups of $\alpha$-CT.

(d) A need for the determination of parameters $\Delta H_{\text {cal }}$ and $\Delta S_{\text {cal }}\left(\Delta S_{m}\right)$ throughout this kind of series, for the proper understanding of atomistic mechanisms behind the stabilizing/destabilizing impact of various additives on proteins, is clearly demonstrated.

\section{Conflict of Interests}

The authors declare that no competing interests exist with any commercial bodies.

\section{Acknowledgments}

Research Grants from The Volkswagen Foundation, Germany (I/83 395), Rustaveli National Science Foundation, Georgia (no. 11/14/2012), and The Deutsche Forschungsgemeinschaft, Germany, are kindly acknowledged. The authors are thankful to Dr. L. Kukutaria for his expert work towards the modification of a DASM-4A instrument and writing the respective PC program. Dr. E. Kiziria is acknowledged for the diverse expert technical support.

\section{References}

[1] C. A. Angell, N. Byrne, and J. Belieres, "Parallel developments in aprotic and protic ionic liquids: physical chemistry and 
applications," Accounts of Chemical Research, vol. 40, no. 11, pp. 1228-1236, 2007.

[2] F. van Rantwijk and R. A. Sheldon, "Biocatalysis in ionic liquids," Chemical Reviews, vol. 107, no. 6, pp. 2757-2785, 2007.

[3] U. A. Rana, P. M. Bayley, R. Vijayaraghavan, P. Howlett, D. R. MacFarlane, and M. Forsyth, "Proton transport in choline dihydrogen phosphate $/ \mathrm{H}_{3} \mathrm{PO}_{4}$ mixtures," Physical Chemistry Chemical Physics, vol. 12, no. 37, pp. 11291-11298, 2010.

[4] A. Kumar and P. Venkatesu, "Overview of the stability of $\alpha$ chymotrypsin in different solvent media," Chemical Reviews, vol. 112, no. 7, pp. 4283-4307, 2012.

[5] T. De Diego, P. Lozano, S. Gmouh, M. Vaultier, and J. L. Iborra, "Fluorescence and CD spectroscopic analysis of the $\alpha$-chymotrypsin stabilization by the ionic liquid, 1-ethyl3-methylimidazolium bis[(trifluoromethyl)sulfonyl]amide," Biotechnology and Bioengineering, vol. 88, no. 7, pp. 916-924, 2004.

[6] K. Fujita, D. R. MacFarlane, and M. Forsyth, "Protein solubilising and stabilising ionic liquids," Chemical Communications, no. 38, pp. 4804-4806, 2005.

[7] K. Fujita and H. Ohno, "Enzymatic activity and thermal stability of metallo proteins in hydrated ionic liquids," Biopolymers, vol. 93, no. 12, pp. 1093-1099, 2010.

[8] N. Byrne and C. A. Angell, "Protein unfolding, and the "tuning in" of reversible intermediate states, in protic ionic liquid media," Journal of Molecular Biology, vol. 378, no. 3, pp. 707-714, 2008.

[9] N. Byrne, J. P. Belieres, and C. A. Angell, "The "refoldability" of selected proteins in ionic liquids as a stabilization criterion, leading to a conjecture on biogenesis," Australian Journal of Chemistry, vol. 62, no. 4, pp. 328-333, 2009.

[10] R. Buchfink, A. Tischer, G. Patil, R. Rudolph, and C. Lange, "Ionic liquids as refolding additives: variation of the anion," Journal of Biotechnology, vol. 150, no. 1, pp. 64-72, 2010.

[11] D. Constatinescu, C. Herrmann, and H. Weingärtner, "Patterns of protein unfolding and protein aggregation in ionic liquids," Physical Chemistry Chemical Physics, vol. 12, no. 8, pp. 17561763, 2010.

[12] P. Attri, P. Venkatesu, A. Kumar, and N. Byrne, "A protic ionic liquid attenuates the deleterious actions of urea on $\alpha$ chymotrypsin," Physical Chemistry Chemical Physics, vol. 13, no. 38, pp. 17023-17026, 2011.

[13] E. Yamamoto, S. Yamaguchi, and T. Nagamune, "Protein refolding by $\mathrm{N}$-alkylpyridinium and n-alkyl-n-methylpyrrolidinium ionic liquids," Applied Biochemistry and Biotechnology, vol. 164, no. 6, pp. 957-967, 2011.

[14] B. Dabirmanesh, S. Daneshjou, A. A. Sepahi et al., "Effect of ionic liquids on the structure, stability and activity of two related $\alpha$-amylases," International Journal of Biological Macromolecules, vol. 48, no. 1, pp. 93-97, 2011.

[15] J. V. Rodrigues, V. Prosinecki, I. Marrucho, L. P. N. Rebelo, and C. M. Gomes, "Protein stability in an ionic liquid milieu: on the use of differential scanning fluorimetry," Physical Chemistry Chemical Physics, vol. 13, no. 30, pp. 13614-13616, 2011.

[16] H. Weingärtner, C. Cabrele, and C. Herrmann, "How ionic liquids can help to stabilize native proteins," Physical Chemistry Chemical Physics, vol. 14, no. 2, pp. 415-426, 2012.

[17] A. M. Figueiredo, J. Sardinha, G. R. Mooreb, and E. J. Cabrita, "Protein destabilisation in ionic liquids: the role of preferential interactions in denaturation," Physical Chemistry and Chemical Physics, vol. 15, pp. 19632-19643, 2013.
[18] E. M. Nordwald and J. L. Kaar, "Mediating electrostatic binding of 1-butyl-3-methylimidazolium chloride to enzyme surfaces improves conformational stability," The Journal of Physical Chemistry B, vol. 117, no. 30, pp. 8977-8986, 2013.

[19] E. M. Nordwald and J. L. Kaar, "Stabilization of enzymes in ionic liquids via modification of enzyme charge," Biotechnology and Bioengineering, vol. 110, no. 9, pp. 2352-2360, 2013.

[20] M. A. M. Latif, B. A. Tejo, R. Abedikargiban, M. B. A. Abdul Rahman, and N. M. Micaêlo, "Modeling stability and flexibility of $\alpha$-Chymotrypsin in room temperature ionic liquids," Journal of Biomolecular Structure and Dynamics, vol. 32, no. 8, pp. 12631273, 2014.

[21] A. Kumar and P. Venkatesu, "Does the stability of proteins in ionic liquids obey the Hofmeister series?" International Journal of Biological Macromolecules, vol. 63, pp. 244-253, 2014.

[22] D. E. Khoshtariya, T. D. Dolidze, T. Tretyakova, D. H. Waldeck, and R. van Eldik, "Electron transfer with azurin at Au/SAM junctions in contact with a protic ionic melt: impact of glassy dynamics," Physical Chemistry and Chemical Physics, vol. 15, pp. 16515-16526, 2013.

[23] D. E. Khoshtariya, M. Shushanian, R. Sujashvili, M. Makharadze, E. Tabuashvili, and G. Getashvili, "Enzymatic activity of $\alpha$-chymotrypsin in the urea-induced moltenglobulelike state: a combined kinetic/ thermodynamic study," Journal of Biological Physics and Chemistry, vol. 3, pp. 2-11, 2003.

[24] M. Shushanyan, R. Sujashvili, E. Tabuashvili, M. Makharadze, G. Getashvili, and D. E. Khoshtariya, "Kinetic and thermodynamic manifestations of thermally induced molten-globulelike state of $\alpha$-chymotrypsin," Journal of Biological Physics and Chemistry, vol. 6, no. 2, pp. 51-55, 2006.

[25] D. E. Khoshtariya, T. D. Dolidze, S. Seifert, D. Sarauli, G. Lee, and R. van Eldik, "Kinetic, thermodynamic, and mechanistic patterns for free (unbound) cytochrome $\mathrm{c}$ at $\mathrm{Au} / \mathrm{SAM}$ junctions: impact of electronic coupling, hydrostatic pressure, and stabilizing/denaturing additives," Chemistry, vol. 12, no. 27, pp. 70417056, 2006.

[26] A. Kumar, P. Attri, and P. Venkatesu, "Effect of polyols on the native structure of $\alpha$-chymotrypsin: a comparable study," Thermochimica Acta, vol. 536, pp. 55-62, 2012.

[27] P. L. Privalov and N. N. Khechinashvilli, "A thermodynamic approach to the problem of stabilization of globular protein structure: a calorimetric study," Journal of Molecular Biology, vol. 86, no. 3, pp. 665-684, 1974.

[28] P. L. Privalov and S. A. Potekhin, "Scanning microcalorimetry in studying temperature-induced changes in proteins," Methods in Enzymology, vol. 131, pp. 4-51, 1986.

[29] E. Freire, W. W. van Osdol, O. L. Mayorga, and J. M. Sanchez-Ruiz, "Calorimetrically determined dynamics of complex unfolding transitions in proteins," Annual Review of Biophysics and Biophysical Chemistry, vol. 19, pp. 159-188, 1990.

[30] T. Tretyakova, M. Shushanyan, T. Partskhaladze, M. Makharadze, R. van Eldik, and D. E. Khoshtariya, "Simplicity within the complexity: bilateral impact of DMSO on the functional and unfolding patterns of $\alpha$-chymotrypsin," Biophysical Chemistry, vol. 175-176, pp. 17-27, 2013.

[31] G. I. Makhatadze and P. L. Privalov, "Energetics of protein structure," Advances in Protein Chemistry, vol. 47, pp. 307-425, 1995.

[32] G. I. Makhatadze and P. L. Privalov, "On the entropy of protein folding," Protein Science, vol. 5, no. 3, pp. 507-510, 1996. 
[33] P. L. Privalov, "Thermodynamic problems in structural molecular biology," Pure and Applied Chemistry, vol. 79, no. 8, pp. 14451462, 2007.

[34] J. Gsponer, H. Hopearuoho, S. B. Whittaker et al., "Determination of an ensemble of structures representing the intermediate state of the bacterial immunity protein Im7," Proceedings of the National Academy of Sciences of the United States of America, vol. 103, no. 1, pp. 99-104, 2006.

[35] C. la Rosa, D. Milardi, D. Grasso, R. Guzzi, and L. Sportelli, "Thermodynamics of the thermal unfolding of azurin," Journal of Physical Chemistry, vol. 99, no. 40, pp. 14864-14870, 1995. 

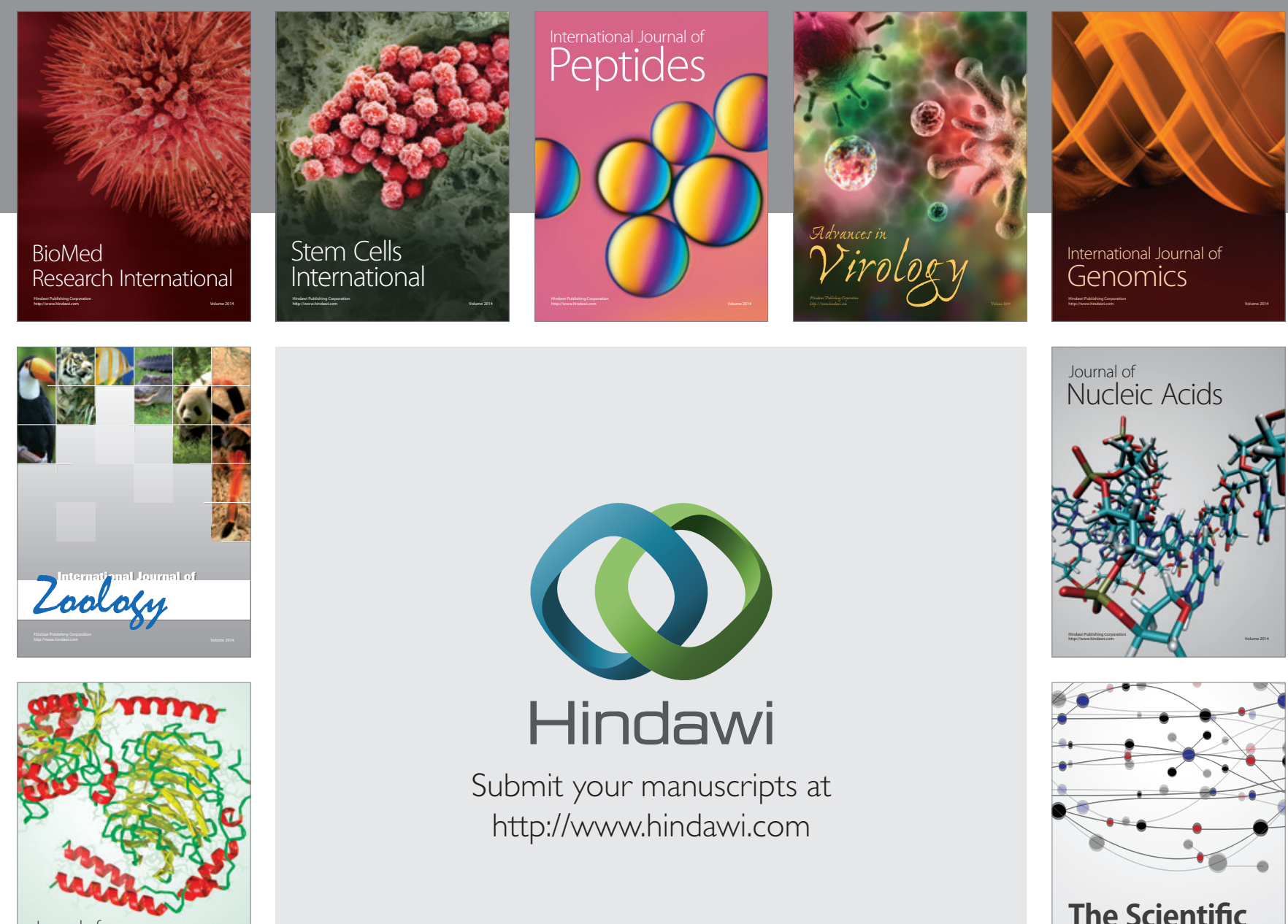

Submit your manuscripts at

http://www.hindawi.com

Journal of
Signal Transduction
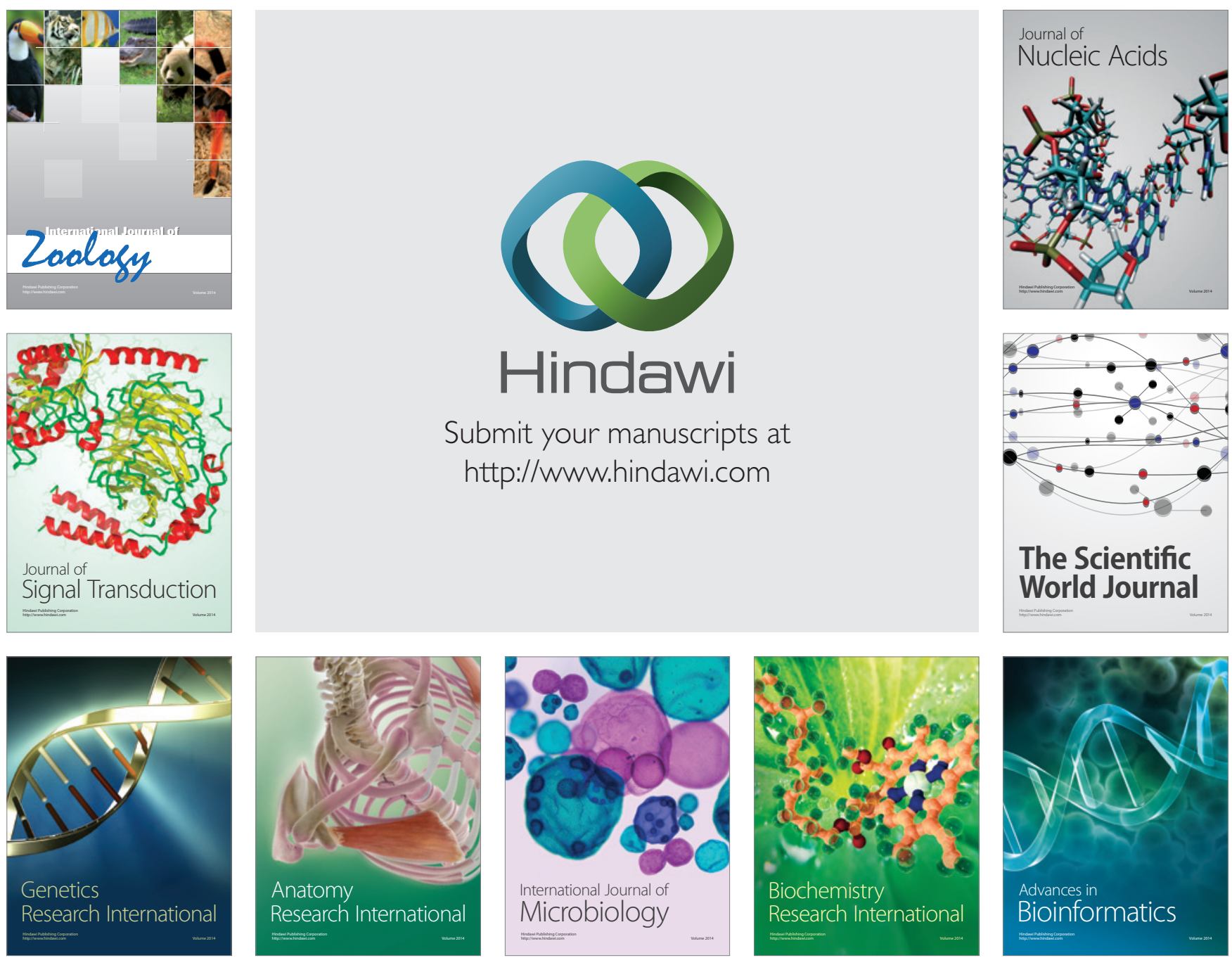

The Scientific World Journal
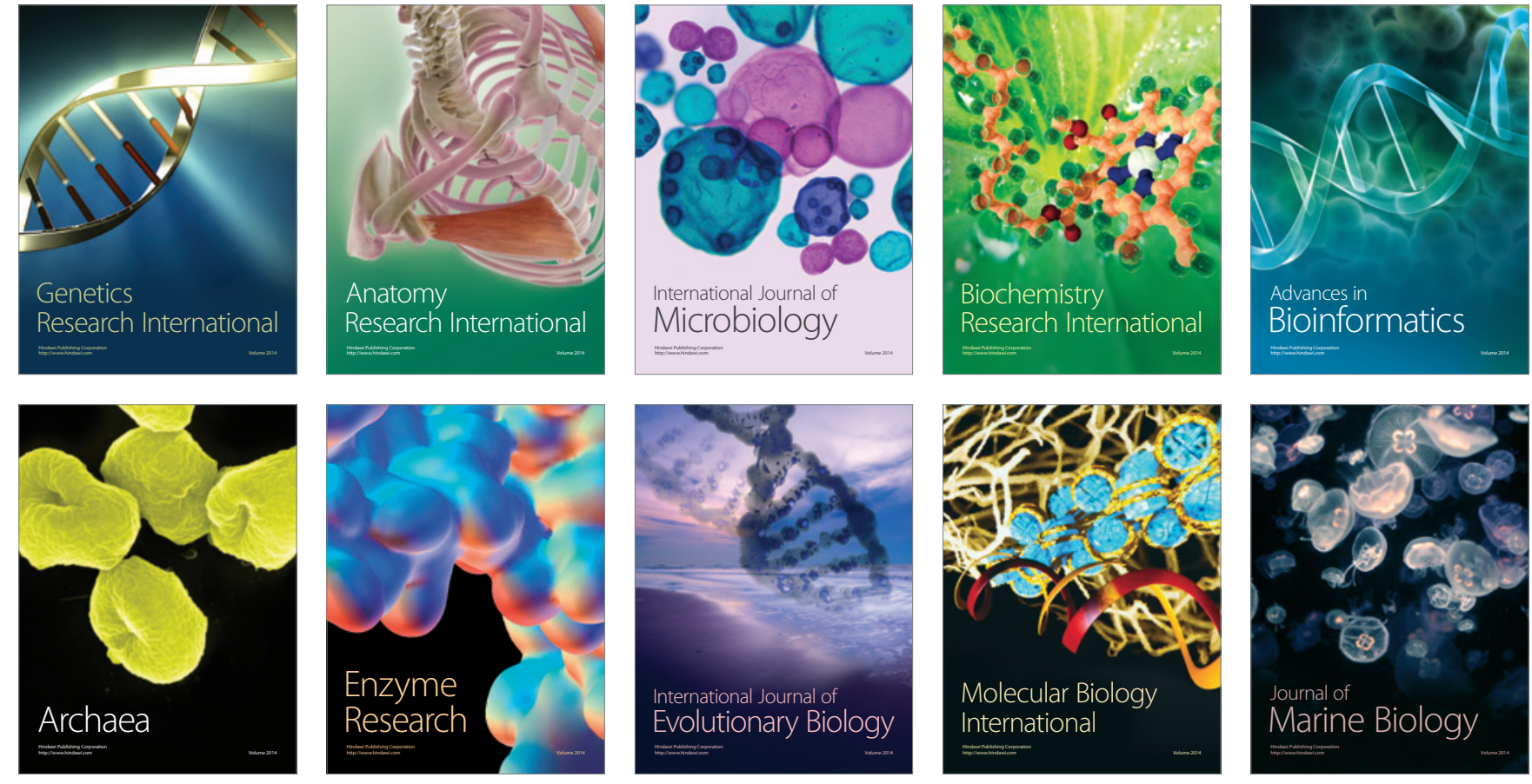